
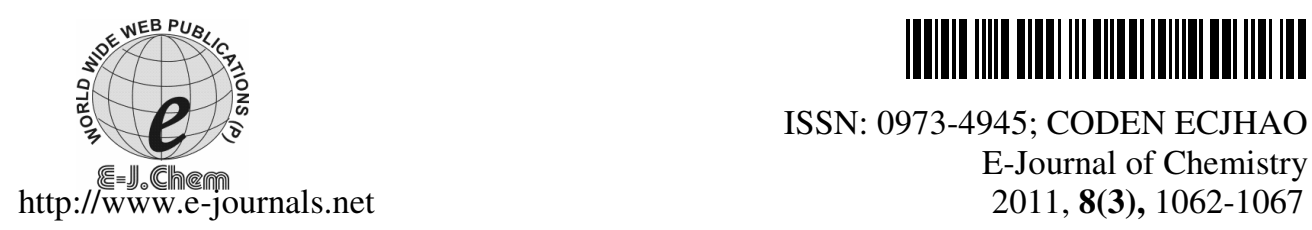

ISSN: 0973-4945; CODEN ECJHAO

E-Journal of Chemistry

2011, 8(3), 1062-1067

\title{
Growth and Characterization of Organic Based Marine Dye NLO Material: 7-Bromo-6-chloro-3-[3-[(2R, 3S)-3-hydroxy-2- piperidyl]-2-oxopropyl]-4(3H)-quinazolinone
}

\author{
M. JAYANDRAN* and V. BALASUBRAMANIAN \\ *Department of Chemistry \\ Mahendra Engineering College, Namakkal-637 503, India \\ Department of Chemistry \\ Amet University, Chennai -603112, India \\ jai_varshi@yahoo.com
}

Received 9 December 2010; Accepted 24 January 2011

\begin{abstract}
An organic marine dye compound (7-bromo-6-chloro-3-[3-[(2R, 3S)3-hydroxy-2-piperidyl]-2-oxopropyl]-4(3H)-quinazolinone) was synthesized. The characteristics of this dye is soluble in water. The organic compound was made into a crystal by slow evaporation technique. The grown crystals were characterized by single crystal $\mathrm{x}$-ray diffraction (XRD) analysis, FTIR studies and UV-visible transmittance studies and the NLO activity of the grown crystal has been checked by second harmonic generation (SHG) test.
\end{abstract}

Keywords: Organic marine dye, Crystal growth, FTIR, XRD, SHG, UV Visible

\section{Introduction}

Nonlinear optical (NLO) materials have gained considerable attention due to their practical applications in the field of optoelectronics ${ }^{1,2}$. The development of NLO materials led to compounds potentially suitable for application in frequency conversion, optical telecommunication, image processing, optical computing and data storage devices ${ }^{3-6}$. Quinazolinone family-type crystals over the years have been subjected to extensive investigation by the researchers for their non-linear optical properties, ${ }^{7,8}$. Among the disulfonic acid, quinoline is the simplest molecule with second harmonic generation efficiency of about one-third of that of the well known $\mathrm{KDP}^{9,10}$, it is expected to get improved NLO properties. Keeping this in mind, the quinoline mixtures have been mixed to form a novel NLO material. 7-Bromo-6-chloro-3-[3-[(2R,3S)-3-hydroxy-2-piperidyl]-2- 
oxopropyl]-4(3H)-quinazolinone reported the details of crystal structure of (Halofuginone) and it is observed from the various properties of crystals report. Hence the aim of this paper is to report the crystal growth of that marine dye and the confirmation has been identified by spectroscopic studies and NLO activity of crystals.

\section{Experimental}

A crystalline compound of halofuginone molecular formula is $\mathrm{C}_{16} \mathrm{H}_{17} \mathrm{BrClN}_{3} \mathrm{O}_{3}$. It belongs to a chemical class of di-azo compound and the structure is shown in Figure 1. The aqueous solution of the dye was prepared prior to the experiments by dissolving the requisite amount of dye in double distilled water at ambient temperature. The substances have a melting point $468.1{ }^{\circ} \mathrm{C}$ and water solubility $209 \mathrm{~g} / \mathrm{L}\left(20^{\circ} \mathrm{C}\right)$

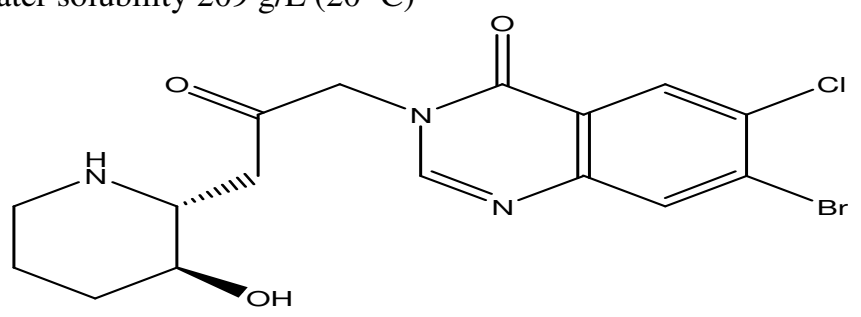

Figure 1. Structure of 7-bromo-6-chloro-3-[3-[(2R, 3S)-3-hydroxy-2-piperidyl]-2-oxopropyl] $4(3 H)$-quinazolinone

\section{Results and Discussion}

\section{XRD analysis}

The grown crystals were subjected to single crystal XRD to confirm the crystalline and also to estimate the lattice parameters by employing Bruker-nonious MACH3/CAD4 single $x$-ray diffractometer. From single crystal $x$-ray diffraction data, it is observed that the 7-bromo-6-chloro-3-[3-[(2R,3S)-3-hydroxy-2-piperidyl]-2-oxopropyl]-4(3H)-quinazolinone. The peaks in the fig show the crystalline nature of halofuginone. Further the peaks are indexed. This is shown in Figure 2.

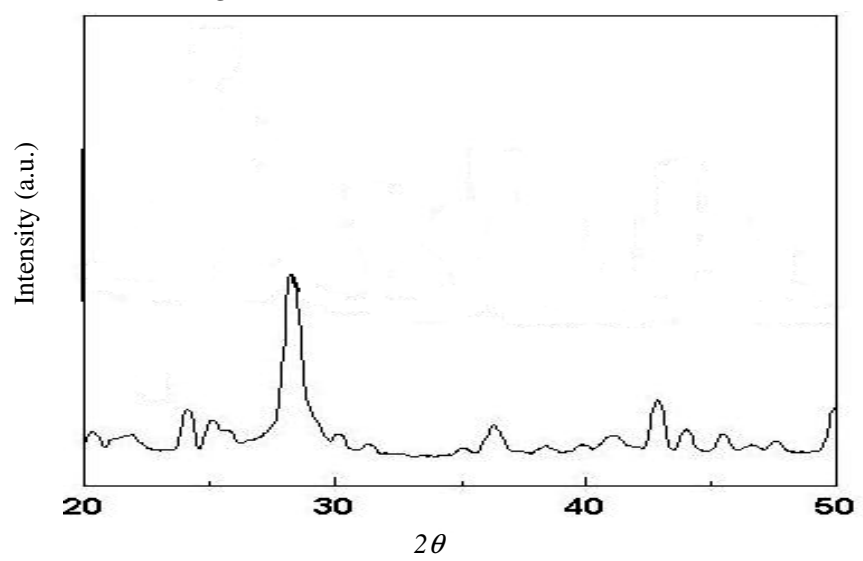

Figure 2. XRD patterns recorded from 7-bromo-6-chloro-3-[3-[(2R,3S)-3-hydroxy-2piperidyl]-2-oxopropyl]-4(3H)-quinazolinone 


\section{FTIR analysis}

The fourier transform infrared (FTIR) spectrum of 7-bromo-6-chloro-3-[3-[(2R,3S)-3hydroxy-2-piperidyl]-2-oxopropyl]-4(3H)-quinazolinone crystal was recorded in the region $400-4000 \mathrm{~cm}^{-1}$ using FTIR SHIMADZU 8400S. Amide functional group appeared at $3300 \mathrm{~cm}^{-1}$ with sharp absorption and also giving $\mathrm{C}=\mathrm{O}$ stretching absorption at $1638 \mathrm{~cm}^{-1}$. It was further confirmed by observing $\mathrm{C}=\mathrm{O}$ overtone absorption appeared at $3810 \mathrm{~cm}^{-1}$ and $3919 \mathrm{~cm}^{-1}$. The strong absorption at $1030 \mathrm{~cm}^{-1}$ was assigned to $\mathrm{C}-\mathrm{O}$ group due to its stretching vibrations. The strong absorption at $1564 \mathrm{~cm}^{-1}$ confirms the presence of $\mathrm{COO}$ functional groups along with the amide group. The asymmetric stretching absorption of $-\mathrm{CH}_{3}$ group was appeared at $2364 \mathrm{~cm}^{-1}$ and its symmetric stretching absorption was appeared at $2224 \mathrm{~cm}^{-1}$. The stretching absorptions in the range from $738 \mathrm{~cm}^{-1}$ confirms the presence of $-\mathrm{Cl}$ group and the another $-\mathrm{Br}$ containing compounds absorb at strongly in the region $576 \mathrm{~cm}^{-1}$. The strong absorption at $3550.32 \mathrm{~cm}^{-1}$ was confirms the presence of $-\mathrm{OH}$ group due to its stretching vibrations. Also, strong absorption at $1411 \mathrm{~cm}^{-1}$ was due to bending absorption of alkyl group. The absorptions were summarized in Table 1 and spectra were given in Figure 3.

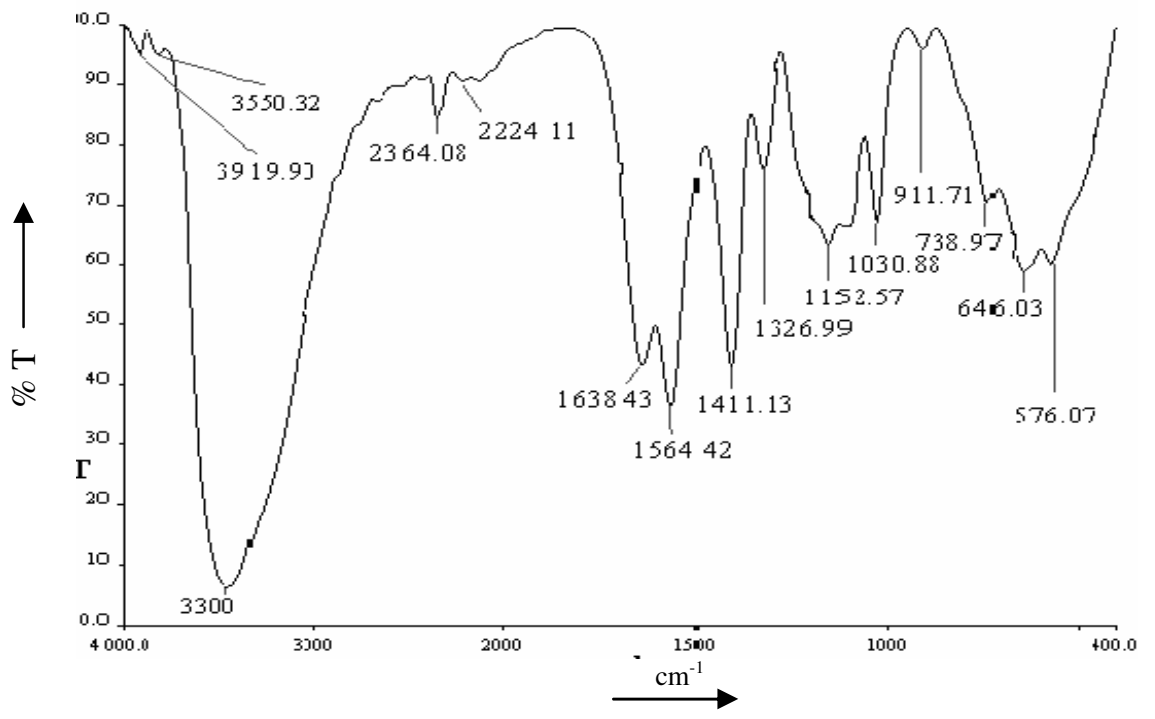

Figure 3. FTIR spectrum of 7-bromo-6-chloro-3-[3-[(2R,3S)-3-hydroxy-2-piperidyl]-2oxopropyl]-4(3H)-quinazolinone crystal

Table 1. Frequencies of the fundamental vibrations of GPC

\begin{tabular}{ccc}
\hline S. No. & Frequency in wavelength, in $\mathrm{cm}^{-1}$ & Assignment of vibration \\
\hline 1 & 3810 and 3919 & $\mathrm{C}=\mathrm{O}$ Overtone absorption \\
2 & 3300 & $-\mathrm{NH}$ stretching vibration \\
3 & 2364 & $\mathrm{sp}^{3}$ asym. Stretching \\
4 & 2224 & $\mathrm{sp}^{3}$ sym. Stretching \\
5 & 1564 & $\mathrm{COO}$ asym. Stretching \\
6 & 1411 & $\mathrm{sp}^{3}$ alkyl bending \\
7 & 3550.32 & $-\mathrm{OH}$ stretching vibration \\
8 & 738 & $-\mathrm{Cl}$ stretching vibration \\
9 & 576 & $-\mathrm{Br}$ stretching vibration \\
\hline
\end{tabular}




\section{Optical transmission spectral analysis}

The UV Visible transmittance spectrum (Figure 4) 7-bromo-6-chloro-3-[3-[(2R, 3S)-3hydroxy-2-piperidyl]-2-oxopropyl]-4(3H)-quinazolinone crystal was recorded in the wavelength range 190-1100 nm, using Lambda 35 spectrometer. Optically polished single crystal of thickness $2 \mathrm{~mm}$ was used for this study. This spectral study may be assisted in understanding electronic structure of the optical band gap of the crystal. The study of the absorption edge is essential in connection with the theory of electronic structure, which leads to the prediction of whether the band structure is affected near the band extreme. It is noticed from the results that 7-bromo-6-chloro-3-[3-[(2R,3S)-3-hydroxy-2-piperidyl]-2oxopropyl]-4(3H)-quinazolinone crystal has transmittance in the entire visible-NIR region 190-2500 $\mathrm{nm}$ of the spectra and the high transmission in the entire visible region and short cut off wavelength facilitates the grown crystals of this work to be potential nonlinear optical materials for second harmonic and third harmonic of Nd: YAG laser. Absorption in the near ultraviolet region arises from electronic transitions associated within the samples. The cut-off wavelength $(\lambda)$ is around $24077 \mathrm{~nm}$. Using the formula $\mathrm{Eg}=\mathrm{hc} / \lambda$, the band energy gap was found to be $6.396 \mathrm{eV}$ have been shown in Figure 4.

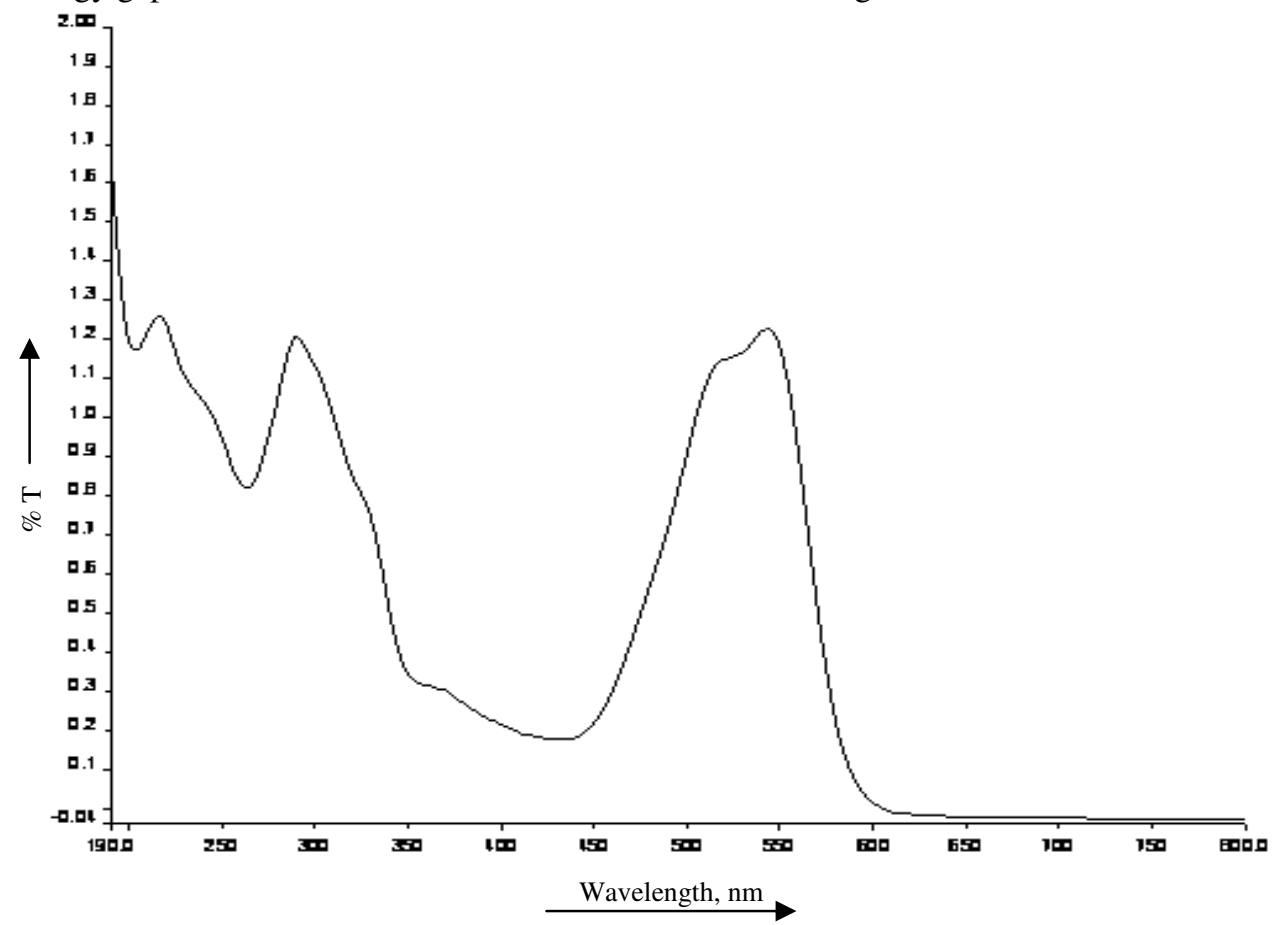

Figure 4. UV-visible transmittance spectrum for 7-bromo-6-chloro-3-[3-[(2R,3S)-3-hydroxy -2-piperidyl]-2-oxopropyl]-4(3H)-quinazolinone crystal

Second harmonic generation (SHG) test

The nonlinear optical (NLO) property of the grown crystal was confirmed by Kurtz-Perry powder technique ${ }^{14}$. The 7-bromo-6-chloro-3-[3-[(2R,3S)-3-hydroxy-2-piperidyl]-2-oxopropyl] $4(3 H)$-quinazolinone crystal was powdered with uniform particle size using a ball mill and it was packed densely between two transparent glass slides. An Nd: YAG laser was used as a light source. This laser device can be operated in two different modes. In the single-shot 
mode, the laser emits an 8 ns pulse. While in the multi-shot mode, the laser produces a continuous train of $8 \mathrm{~ns}$ pulse at a repetition rate of $10 \mathrm{~Hz}$. In the present study, a multishot mode of $8 \mathrm{~ns}$ laser pulse with a spot radius of $1 \mathrm{~mm}$ was used. The experimental setup for measuring SHG efficiency is shown in the Figure 5. A fundamental laser beam of $1064 \mathrm{~nm}$ wavelength, $8 \mathrm{~ns}$ pulse with $10 \mathrm{~Hz}$ pulse rate was made to fall normally on the sample cell(S). The power of the incident beam was measured using a power meter. The filter F1 removes the $1064 \mathrm{~nm}$ light and the filter F2 is a BG-38 filter, which also removes the residual $1064 \mathrm{~nm}$ light. F3 is an interference filter with bandwidth of $4 \mathrm{~nm}$ and central wavelength $532 \mathrm{~nm}$. The green light was detected by a photomultiplier tube (PMT) and displayed on a cathode ray oscilloscope(CRO). KDP crystal was powdered into identical size as 7-bromo-6-chloro-3-[3-[(2R, 3S)-3-hydroxy-2-piperidyl]-2-oxopropyl]-4(3H)quinazolinone crystal and it was used as reference material in the SHG measurement. In the NLO process that taking place in the sample, it converts the $1064 \mathrm{~nm}$ radiation into green light $(\lambda=532 \mathrm{~nm})$ when Nd: YAG laser light is passed into the sample and this confirms the SHG. It was found that the efficiency of SHG is 0.76 times that of the standard KDP.

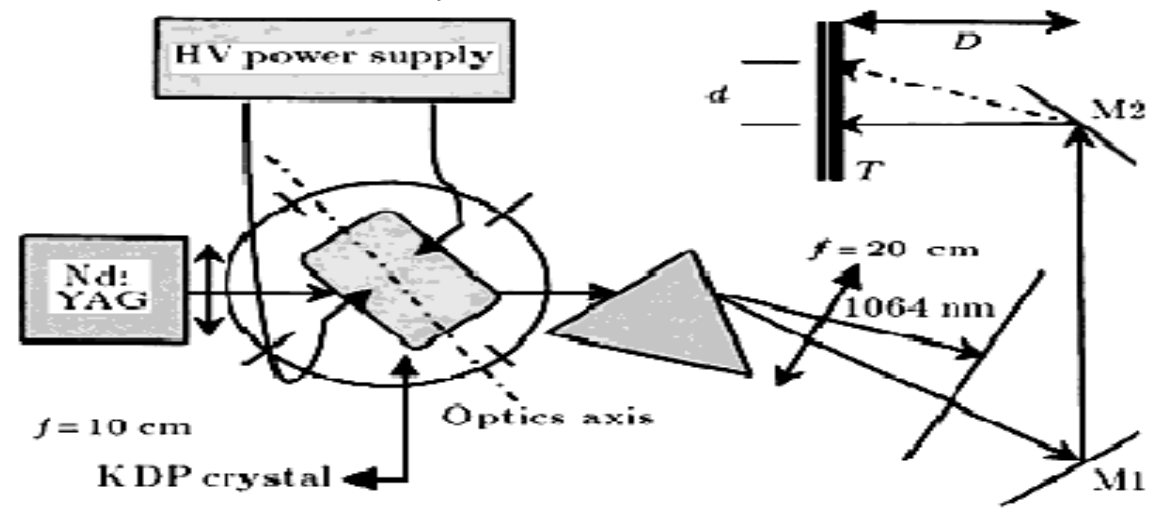

Figure 5. Experimental setup for SHG measurement

\section{Conclusion}

The crystals of 7-bromo-6-chloro-3-[3-[(2R, 3S)-3-hydroxy-2-piperidyl]-2-oxopropyl]$4(3 H)$-quinazolinone was grown by slow evaporation at room temperature. The grown crystal was characterized by using x-ray diffraction method. The quality of the crystal examined by high resolution x-ray diffraction study indicates that the crystalline quality which reasonably good withouthaving any internal structural grain boundaries. Functional groups and the modes of vibrations were identified by FTIR spectroscopy. The grown crystal is transparent in the entire visible region. The SHG efficiency was determined as $15 \%$ of that of the standard KDP. The UV visible-NIR spectrum of the 7-bromo-6-chloro-3[3-[(2R, 3S)-3-hydroxy-2-piperidyl]-2-oxopropyl]-4(3H)-quinazolinone showed that the crystal is transparent in the range of 190-2500 $\mathrm{nm}$. The band gap energy of that compound has been estimated from the optical transmittance spectrum is about $2.24 \mathrm{eV}$. The NLO crystal of 403 efficiency tested by high intensity Nd: YAG laser as a source is about 60 times greater than that of KDP.

\section{Acknowledgment}

The authors are thankful to Mahendra Engineering College and AMET University to do this work and Mr. P.Vasudevan for his suggestion to complete this work. 


\section{References}

1. Wang X Q, Xu D, Lu M, Yuan D, J Huang J, Cheng X, Xie T, Zhang G H, S.L. Wang S L, Guo S Y, Liu J R, Yang Z H and Wang P, J Crystal Growth, 2002, 234, 469.

2. Duan X L, Yuan D R, Wang X Q, Guo S Y, Zhang J G, Xu D and Lu M K, Cryst Res Technol., 2002, 37, 1066-1074.

3. Frazier C C, Cockerhamn M P, Chauchard E A and Lee C H, J Opt Soc Am B, 1987, 4, 1899.

4. Monaco S B, Davis L E, Velsko S P, Wong F T and Eimerl D, J Crystal Growth, 1987, 85, 252.

5. Gunter P, Bosshard Ch, Sutter K, Arend H, Chapuis G, Twieg R J and Dobrowolski D, Appl Phys Lett., 1997, 50, 486-488.

6. Krishnan C, Selvarajan P and Freeda T H, Mat Lett., 2008, 629, 4414.

7. Kitazawa M, Higuchi R and Takahashi M, Appl Phys Lett., 1994, 64, 2477.

8. Misoguti L, Varela A T, Nunes F D, Bagnato V S, Melo F E A, Mendes Filho J and Zilio S C, Opt Mater., 1996, 6, 147.

9. Suresh Kumar B, Sudarsana Kumar M R and Rajendra Babu K, Cryst Res Technol., 2008, 43, 745-750.

10. Razzetti C, Ardoino M, Zanotti L, Zha M and Paorici C, Cryst Res Technol., 2002, 37, 456-465. 


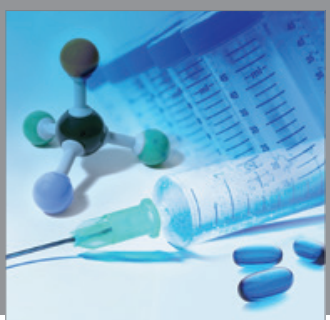

International Journal of

Medicinal Chemistry

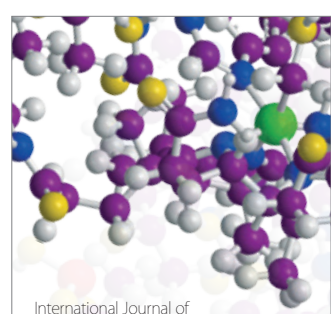

Carbohydrate Chemistry

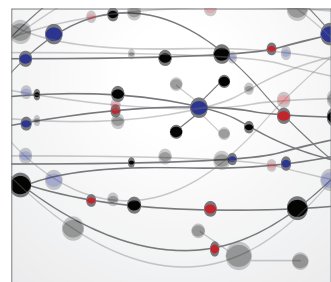

The Scientific World Journal
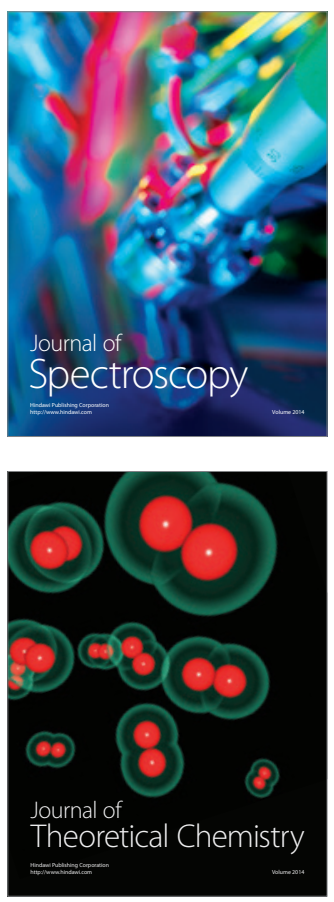
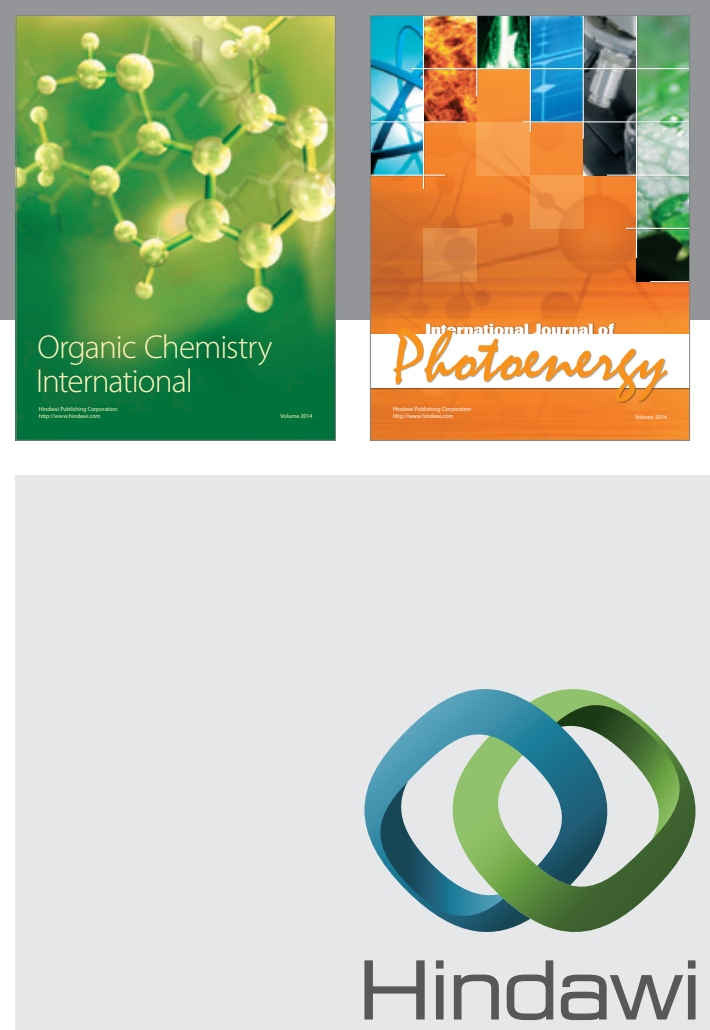

Submit your manuscripts at

http://www.hindawi.com
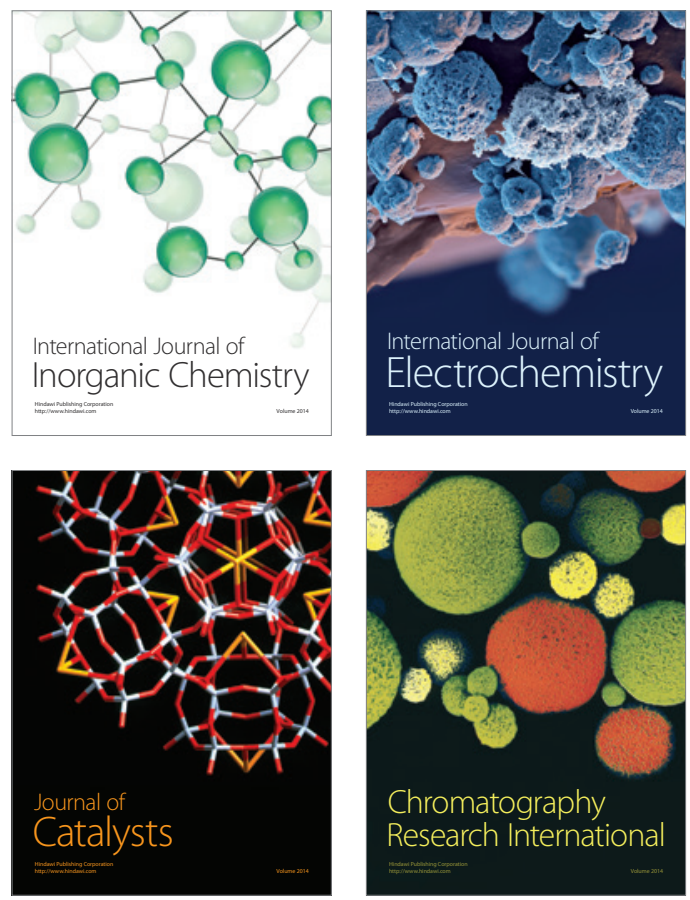
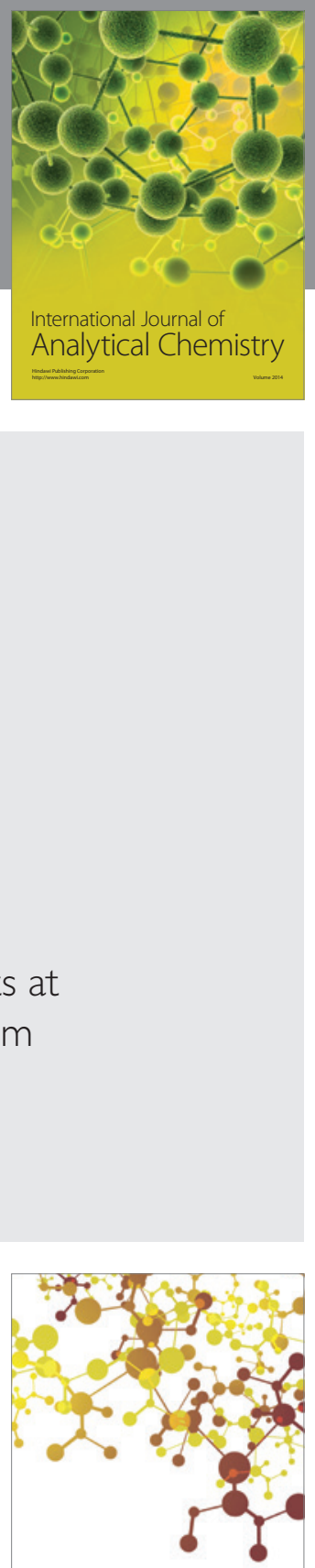

Journal of

Applied Chemistry
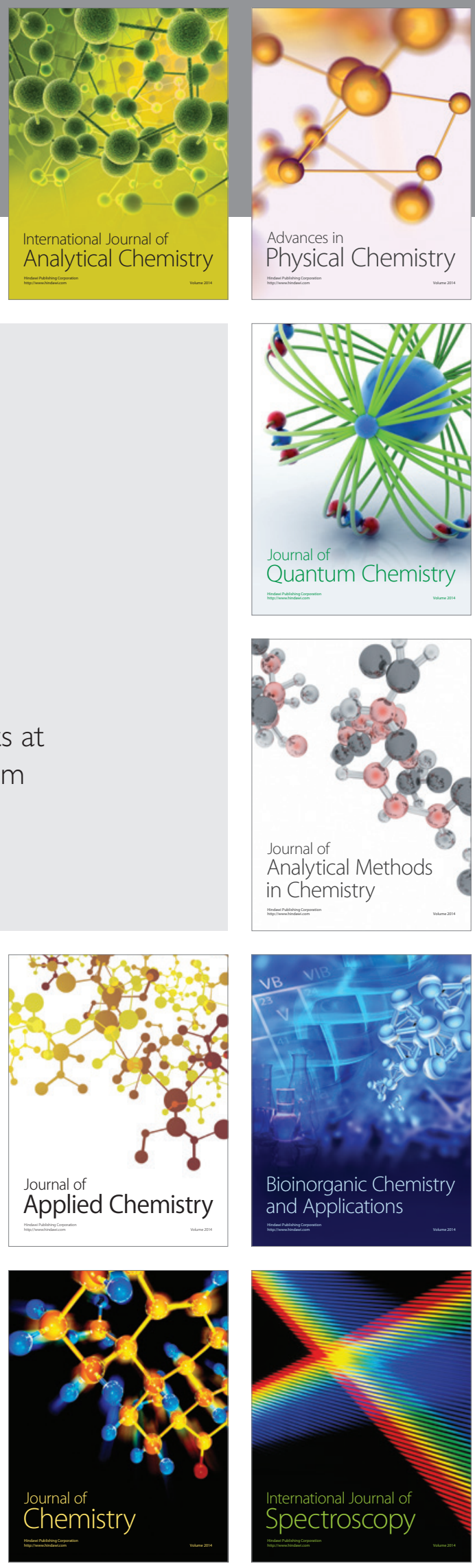